\title{
Prevalence and associations of small for gestational age in a cohort of women birthing in Honiara, the Solomon Islands.
}

Kirsten Black ( $\nabla$ kirsten.black@sydney.edu.au )

University of Sydney Sydney Medical School https://orcid.org/0000-0003-0030-2431

Manarangi De Silva

The University of Melbourne School of Historical and Philosophical Studies

Kim Duckyeon

The University of Sydney Sydney Law School

Hannah Klucknow

John Hunter Hospital

Susan Philipa Walker

The University of Melbourne

Leeanne Rose Panisi

Solomon Islands Ministry of Health

Research article

Keywords: Small for gestational age (SGA), neonatal and child morbidity, antenatal care

Posted Date: December 9th, 2019

DOI: https://doi.org/10.21203/rs.2.18515/v1

License: (9) (i) This work is licensed under a Creative Commons Attribution 4.0 International License.

Read Full License 


\section{Abstract}

Background: Small for gestational age (SGA) is a significant indicator of subsequent neonatal and child morbidity. In low- and middle-income countries (LMICs), around a fifth of infants are born small for gestational age as defined by the Intergrowth-21 st (IG21) standard. We sought to examine the prevalence and associations of small for gestational age using IG21 among women attending antenatal care in Honiara, Solomon Islands, to compare these rates with regional data and explore the demographic factors associated with this outcome.

Methods: From January 2014 - April 2015 we undertook a prospective cohort study that used a structured questionnaire to collect data on women presenting to the National Referral Hospital and community clinics in Honiara for antenatal care. Follow up data was collected by midwives regarding the outcomes of these pregnancies. We used the IG21 data to derive birthweight centiles.

Results: Of the 1441 women enrolled, 1311 records on singleton live births were obtained. Gestational age was calculated on a recorded last menstrual period in 1058 (80.7\%) and relied on clinical assessment at birth in 253 (19.3\%). Using IG21 the prevalence of SGA in this population was $16.8 \%$ with the majority being term SGA. SGA was significantly associated with maternal primiparity and preeclampsia, and SGA babies had higher rates of delivery by caesarean section and neonatal nursery admission.

Conclusions: The rate of SGA in this study was a little lower than pooled estimates across all LMICs. Improving antenatal care attendance, access to ultrasound assessment of gestational age and employing birth growth standards will assist to monitor the prevalence of SGA and manage the consequences.

\section{Background}

Being born small for gestational age (ie. below the $10^{\text {th }}$ centile of a birthweight-for-gestational-age, gender-specific reference population [1]) is a leading risk factor for stillbirth as well as neonatal mortality and morbidity globally. An estimated 23 million babies are born small for gestational age (SGA) each year, mostly in low and middle income countries (LMICs) [2] [3] with the highest rates reported in South Asia $[4][3,5]$. In the short term, SGA fetuses carry a 3-4-fold increased risk of stillbirth. Infants born SGA have increased mortality and morbidity in the neonatal period and beyond as they are more likely to have neonatal infections, perinatal respiratory distress syndrome, jaundice, polycythemia, hypoglycemia, poor feeding, and hypothermia $[1,3,6]$. Furthermore, infants born SGA are at higher risk of long-term adverse health outcomes, such as coronary artery disease, stroke, hypertension and type 2 diabetes [7]. Improving these short- and long-term outcomes requires improved diagnosis of SGA, so that appropriate surveillance and management strategies are in place.

A major challenge in diagnosing SGA, and thus assessing its impact, is selecting an appropriate reference range with which to apply the $10^{\text {th }}$ centile cut-off. While population specific reference standards might be intuitively considered the most appropriate for LMICs, there is a risk that just assigning the bottom $10 \%$ of 
liveborn infants as SGA may underestimate the true burden of perinatal disadvantage. In an effort to overcome this, several consortia have established international optimal fetal growth standards that could be universally applied. These standards may perform better in identifying infants at greatest risk. One such standard is Intergrowth-2 $1^{\text {st }}$ project (IG21) [8] that established the first international, multiethnic standard from eight geographically defined populations that enables a common single standard to describe optimal and aspirational fetal growth around the world [9]. IG21 deliberately enrolled a cohort of women at low risk of perinatal disadvantage: they didn't smoke, were well nourished, were not hypertensive and had access to good antenatal care. Thus, IG21 sets 'aspirational targets' of fetal growth/ birthweight in optimal populations, describing fetuses as they should grow, not how they do. The IG21 study found that among healthy pregnant women with adequate nutrition, fetal growth was comparable across different populations globally, suggesting that ethnic differences in fetal growth were more related to deprivation than geography [10].

The Solomon Islands is an archipelago nation of around 600,000 people consisting of six major islands and over 900 small islands. On the Human Development Index, the country is representative of LMICs in the Western Pacific. Currently no birth growth charts are routinely in use apart from the WHO infant growth charts which provide centiles only for term infants. In this study we have used IG21 to describe the burden of suboptimal fetal growth in a cohort of women attending for antenatal care at the Honiara City Council (HCC) clinics and at the National Referral Hospital in Honiara.

\section{Methods}

The National Referral Hospital is on the main island Guadalcanal, manages 5000-6000 deliveries annually and is supported by nine surrounding HCC clinics in Guadalcanal province. We undertook a prospective cohort study of women presenting for antenatal care to these clinics between January 2014 and May 2015. Research midwives attended HCC clinics on random days to recruit women at their booking or second visit. Written or verbal (with thumbprint) consent was obtained from all participants after verbal and/or written information was provided in English or Melanesian Pidgin. All women were over the age of 18 and consented to participation.

At the time of recruitment, comprehensive perinatal data collection was prospectively gathered in a range of domains. Those relevant to this study included background and socio-demographic factors, including age, marital status, education and employment status and information on reproductive history, including age at first intercourse, previous pregnancies including their history of livebirths and perinatal deaths. We collected birth outcomes including obstetric history, past pregnancies, medical conditions, pregnancy complications, details about the onset of labour (induction, analgesia, duration), delivery (anesthesia, complications) and neonatal details including date of birth, estimated gestation, mode of delivery, gender, birthweight, live or stillborn, length, head circumference, need for resuscitation measures, Apgar scores and neonatal nursery admission. Preeclampsia was defined by local standards as the presence of high blood pressure above 140/90 on two occasions with the presence proteinuria. Data collection was undertaken by only 2 trained local female midwives in an effort to reduce sources of bias. 
Where possible gestational age was calculated on a recorded last menstrual period (LMP). Where the LMP was not available, the gestational age was based on the clinical estimate of age of the neonate at birth and for these pregnancies we took the midpoint of the estimated week, eg. 37 weeks and 3 days, and then converted it to days as is required by IG21. An IG birthweight centile was thus generated for each infant. In 121 women (1.6\%) no birth data were available.

Ethics approval was obtained from the University of Sydney Human Research Ethics (2014/096) and from the National Health Research and Ethics Committee of the Solomon Islands Ministry of Health and Medical Services (HRC13/13).

\section{Statistical Analysis}

All analysis was performed using the statistical software STATA (version 13.1, StataCorp, College Station, TX, USA). Preterm birth (PTB) was defined as birth before 37th week of pregnancy. Small for gestational age (SGA) was defined according to the separate male and female centile charts from the IG21 st project [8], using measured gestational age and birth weights. Stillbirths were excluded from analysis.

Where data for baseline characteristics were missing, we reported the number of available observations. All baseline variables were categorical, and were reported as frequency (percentage), and compared using chi-squared test. All tests were pre-planned to be two-sided with significance level $a=0.05$. Adjustments for multiple comparisons were not undertaken. Predictors of SGA were modelled using multiple logistic regression analysis. Collinearity and interaction effects were examined and confirmed to be absent. Goodness of fit of the final multivariate logistic models was assessed using the Hosmer-Lemeshow test, whose $\mathrm{p}$-values exceeded 0.05 .

\section{Results}

Prospective birth data from the National Referral Hospital and HCC clinics were obtained on 1,332 of the 1441 women recruited. Twelve women in this cohort had a stillbirth (these weights were not recorded) and there were 9 women with multiple pregnancies that were excluded from the analysis. Therefore, the analysis is based on 1311 births (Figure 1). Gestational age was calculated on a recorded LMP in 1058 $(80.7 \%)$ and in 253 women (19.3\%) the gestational age was based on the clinical estimate of gestational age at birth because the LMP was not known.

Table 1 demonstrates the maternal characteristics of the sample. The majority of women were aged between $20-34$ years and $41 \%$ were primiparous. Pre-eclampsia occurred in $3 \%$ of women.

The mean IG birthweight centile for all births was 43.6 (SD 29.97). The prevalence of small for gestational age was $220 / 1311(17 \%)$ and the proportion of all centile groups is shown in Table 2 . Of the 220 babies less than the $10^{\text {th }}$ centile, $94(43 \%)$ were under the $3^{\text {rd }}$ centile and $44(20 \%)$ were under the $1^{\text {st }}$. Most of the SGA occurred in term infants. Only 5 of the 170 live births considered preterm (4.5\%) were SGA. 
Table 3 documents the univariate and multivariable associations of SGA and maternal characteristics. There were positive associations with SGA and maternal age less than 20 years, primiparity and preeclampsia in univariate analysis. After adjusting for maternal age in multivariable analysis, primiparity and preeclampsia remained significantly associated with SGA.

SGA was associated with a range of adverse neonatal outcomes including Apgar less than 7 at 5 minutes with $2.3 \%$ (5) of SGA babies recorded with a low Apgar compared to $0.7 \%$ (8) of those that were appropriate for gestational age (AGA) $(p=0.036)$. SGA babies were significantly more likely to be born via elective or emergency caesarean section compared to those that were AGA $(6.5 \%$ versus $2.4 \% ; p=0.013)$.

\section{Discussion}

This is a novel study which examines SGA in the Solomon Islands using the IG21 standard [8] charts over a period of 18 months. The prevalence of SGA of $16.8 \%$ is closely aligned with regional estimates from Oceania of $16 \%$ [4]. In addition, nearly $30 \%$ of the babies were below the $20^{\text {th }}$ centile, which still confers increased risks of adverse outcomes related to uteroplacental insufficiency including stillbirth, neonatal death, acidosis, admission to nursery and respiratory distress syndrome. We found a significant association between SGA and primiparity and preeclampsia. The relationship between maternal blood pressure and fetal weight is well documented, with preeclampsia affecting uteroplacental development and leading directly to growth restriction $[11,12]$ Primiparity is consistently associated with SGA in studies in both high $[13,14]$ and low income settings with pre-pregnancy weight, nutrition and increased risks of placental disorders such as preeclampsia likely playing a role[15, 16].

The co-occurrence of prematurity and SGA places infants at substantially, and potentially synergistically, higher risk of morbidity and mortality compared with infants who experience prematurity or SGA alone. The majority of SGA babies in our study were term and this aligns with the 2017 Child Epidemiology Reference Group study across 14 sites in LMICs that noted only 1.5 million of the 23.3 million SGA infants were preterm (6.4\%) [4]. Nevertheless, one of the limitations of studies such as this is that small babies in poorly dated pregnancies may be more likely to be classified as premature but appropriately grown, rather than growth restricted.

One of the ongoing obstacles for research and programmatic projects in LMICs such as the Solomon Islands is obtaining accurate dating of gestational age. Currently the WHO recommends the routine use of antenatal ultrasound to ascertain estimated gestational age, ideally prior to 24 weeks $[17,18]$ but this is currently difficult to achieve in the Solomon Islands with many women booking late and a lack of equipment and skilled personnel to perform basic obstetric ultrasound outside of the hospital-setting. As a result, less accurate measures, such as last menstrual period, symphysio-fundal height, postnatal examination of the baby or birthweight are commonly utilised in LMICs [1].

The causes of SGA in the Solomon Islands are varied, but major contributors include poor antenatal care, malaria and malnutrition $[7,19,20]$. There are significant geographical and economic barriers to 
accessing quality and timely antenatal care in the Solomon Islands, with many women booking late and attending much fewer than the WHO recommended 8 visits to reduce perinatal morbidity and mortality. There is poor health awareness amongst many women around the importance of early, and frequent, antenatal visits. Currently, it is recommended that women receive malaria prophylaxis during the antenatal period [7], however compliance with this is difficult to measure and relies on antenatal attendance. Furthermore, this relies on adequate supply of anti-malarial prophylaxis, which is often inconsistent in the community setting. Anaemia in pregnant women is highly prevalent with recent World Bank data [21] reporting that $48.6 \%$ of pregnant women in the Solomon Islands are anaemic, compared with only $15 \%$ of pregnant women in developed countries, mainly secondary to poverty, chronic worm infection, recurrent malaria and dengue infection. Malnutrition is another modifiable risk factor which is extremely common in the Pacific due to a lack of access to affordable fresh, nutritious food, also contributing to anaemia [7].

Our study highlights the excess burden of SGA shouldered by LMIC such as the Solomon Islands, and the urgent need to strengthen public health and education promoting early and adequate antenatal care in order to reduce the incidence of SGA. This should be supported by improved resources and health worker training to enable delivery of routine antenatal ultrasounds which could improve gestational age calculation, as well as detection and management of SGA. Furthermore, although our study did not investigate anaemia, nutrition and malaria infection specifically, public health measures to strengthen the quality of antenatal care to improve the detection and treatment of these important factors should also be addressed when tackling SGA and preterm birth.

\section{Limitations}

There are several limitations of this study. The first is that the gestational estimates were not validated by ultrasound scan due to resource barriers, which limits the accuracy of our estimations of SGA, although most pregnancies were dated according to reliable menstrual data. While we used standardised definition for conditions such as preeclampsia, there remains potential for classification error. Furthermore, we did not collect critical data on a range of factors which are known to be associated with SGA, such as maternal anaemia, maternal malaria, maternal stunting and maternal diet. Additionally, whilst this is the largest study of this kind in the Solomon Islands to date, these data were collected from mothers on the main island of Guadalcanal only, and thus may not be representative of women living elsewhere. Finally, our analyses only represented the burden of SGA among live born babies. Intrauterine growth restriction is an important cause of stillbirth, and not represented in this analysis.

\section{Conclusion}

This study has confirmed that rates of SGA based on IG21 in the Solomon Islands are comparable to regional estimates with the strongest maternal predictors being primiparity and pre-eclampsia. There needs to be a strong investment in improving fetal growth through reducing smoking, malaria infection 
and improving nutrition, as well as improving antenatal attendance. Importantly, future research in this area will require accurate and feasible methods of gestational age dating to improve our epidemiologic understanding of the true burden of SGA.

\section{List Of Abbreviations}

LMIC - Low and low-to-middle income country

SGA - Small for gestational age

IG21 - Intergrowth 21

HCC - Honiara City Council

\section{Declarations}

\section{Ethics approval and consent to participate}

Ethics approval was obtained from the University of Sydney Human Research Ethics (2014/096) and from the National Health Research and Ethics Committee of the Solomon Islands Ministry of Health and Medical Services (HRC13/13).

All women consented to participation.

\section{Consent for publication}

There is no individual data used in this manuscript in any form, therefore consent for publication was not necessary.

\section{Availability of data and materials}

The datasets used and/or analysed during the current study are available from the corresponding author on reasonable request.

\section{Competing interests}

The authors declare that there are no competing interests.

\section{Funding}

There was no additional funding for this study.

\section{Authors contributions}

LP and KB collected the data. KB HK and KD analyzed patient data. KB, MD and SP interpreted the analyzed data and contributed to writing the manuscript. All authors read and approved the final manuscript. 


\section{Acknowledgements}

The authors would like to acknowledge Anna Jatobatu and Jessie Larui for their invaluable assistance in collecting patient data for the study.

\section{References}

1.Lee, A. C. C., et al., National and regional estimates of term and preterm babies born small for gestational age in 138 low-income and middle-income countries in 2010. The Lancet Global Health, 2013. 1(1): p. e26-e36.

2.Black, R. E., Global Prevalence of Small for Gestational Age Births. Nestle Nutr Inst Workshop Ser, 2015. 81:p. 1-7.

3.Every Newborn: An action plan to end preventable deaths U. World Health Organizartion, Editor. 2014.

4.Lee, A. C., et al., Estimates of burden and consequences of infants born small for gestational age in low and middle income countries with INTERGROWTH-21(st) standard: analysis of CHERG datasets. BMJ (Clinical research ed.), 2017. 358: p. j3677-j3677.

5.Chang, A. L., et al., Maternal risk factors and perinatal outcomes among pacific islander groups in Hawaii: a retrospective cohort study using statewide hospital data. BMC Pregnancy and Childbirth, 2015. 15(1).

6.Alkema, L., et al., Global, regional, and national levels and trends in maternal mortality between 1990 and 2015, with scenario-based projections to 2030: a systematic analysis by the UN Maternal Mortality Estimation Inter-Agency Group. The Lancet, 2016. 387(10017): p. 462-474.

7.Cates, J. E., et al., Malaria, malnutrition, and birthweight: A meta-analysis using individual participant data. PLoS Med, 2017. 14(8): p. e1002373.

8.Papageorghiou, A. T., et al., The INTERGROWTH-21 st fetal growth standards: toward the global integration of pregnancy and pediatric care. American Journal of Obstetrics and Gynecology, 2018. 218(2): p. S630-S640.

9.Villar, J., et al., The objectives, design and implementation of the INTERGROWTH-21st Project. BJOG: An International Journal of Obstetrics \& Gynaecology, 2013. 120(s2): p. 9-26.

10.Cheikh Ismail, L., et al., Anthropometric standardisation and quality control protocols for the construction of new, international, fetal and newborn growth standards: the INTERGROWTH-21st Project. BJOG: An International Journal of Obstetrics \& Gynaecology, 2013. 120(s2): p. 48-55.

11.Valero de Bernabé, J., et al., Risk factors for low birth weight: a review. European Journal of Obstetrics \& Gynecology and Reproductive Biology, 2004. 116(1): p. 3-15. 
12.Muhwava, L. S., N. Morojele, and L. London, Psychosocial factors associated with early initiation and frequency of antenatal care (ANC) visits in a rural and urban setting in South Africa: a cross-sectional survey. BMC Pregnancy and Childbirth, 2016. 16(18).

13.Spinillo, A., et al., Interaction between risk factors for fetal growth retardation associated with abnormal umbilical artery Doppler studies. Acta Obstet Gynecol Scand, 2004. 83(5): p. 431-5.

14.Campbell, M. K., et al., Determinants of small for gestational age birth at term. Paediatr Perinat Epidemiol, 2012. 26(6): p. 525-33.

15.Gao, W., et al., Risk factors for preterm and small-for-gestational-age babies: A cohort from the Pacific Islands Families Study. Journal of Paediatrics and Child Health, 2006. 42(12): p. 785-792.

16.McCowan, L. and R. P. Horgan, Risk factors for small for gestational age infants. Best Practice \& Research Clinical Obstetrics \& Gynaecology, 2009. 23(6): p. 779-793.

17.Obesity Australia, Obesity: A National Epidemic and its Impact on Australia 2014, Obesity Australia.

18.Ma, R. C. W., et al., Clinical management of pregnancy in the obese mother: before conception, during pregnancy, and post partum. The Lancet Diabetes \& Endocrinology, 2016. 4(12): p. 1037-1049.

19.Launiala, A. and T. Kulmala, The importance of understanding the local context: women's perceptions and knowledge concerning malaria in pregnancy in rural Malawi. Acta Trop, 2006. 98(2): p. 111-7.

20.Unger, H. W., et al., Factors associated with ultrasound-aided detection of suboptimal fetal growth in a malaria-endemic area in Papua New Guinea. BMC Pregnancy Childbirth, 2015. 15: p. 83.

21. World Development Indicators, World Bank. 2016.

\section{Tables}

Table 1. Maternal characteristics 


\begin{tabular}{ll}
\hline Maternal Characteristics & Number (\%) \\
\hline Maternal age group & $142(10.9 \%)$ \\
$<20$ & $750(57.4 \%)$ \\
$20-29$ & $281(21.5 \%)$ \\
$30-34$ & $133(10.2 \%)$ \\
$\geq 35$ & \\
Education & $138(10.5 \%)$ \\
Tertiary & $683(52.1 \%)$ \\
Secondary & $404(31.0 \%)$ \\
Primary & $77(5.9 \%)$ \\
$\quad$ None & \\
Marital status & $1171(90.0)$ \\
Married/defacto & $132(10.0 \%)$ \\
Single/divorced/widowed & \\
Employment & $301(23.2 \%)$ \\
Employed & $895(69.1 \%)$ \\
Unemployed & $99(7.6)$ \\
Student & \\
Literacy & $84(6.5)$ \\
illiterate & $419(32.7)$ \\
Reads with difficulty & $780(60.8)$ \\
Reads easily & \\
Smoking status during pregnancy &
\end{tabular}

Table 2. Weight centiles according to Intergrowth 21 study.

\begin{tabular}{lll}
\hline Centile range & Numbers & Percentage \\
\hline$<_{10}^{\text {th }}$ & 220 & 16.8 \\
10-20th & 146 & 11.1 \\
20-30th & 139 & 10.6 \\
30-40th & 279 & 21.3 \\
50-60th & 112 & 8.5 \\
60-70th & 118 & 9.0 \\
70-80th & 80 & 6.1 \\
80-90th & 87 & 6.6 \\
90-100th & 130 & 9.9 \\
\hline
\end{tabular}


Table 3. Univariate and multivariate maternal associations with small for gestational age (SGA)

\begin{tabular}{|c|c|c|c|c|c|}
\hline Variable & Total & SGA (\%) & P-value & $\begin{array}{l}\text { Adjusted OR for SGA } \\
(95 \% \mathrm{CI}) *\end{array}$ & P-value \\
\hline \multicolumn{6}{|l|}{ Age group } \\
\hline$<20$ & 143 & $31(21.7)$ & 0.017 & 1 & \\
\hline $20-29$ & 781 & $136(18.1)$ & & $1.061(0.670-1.681)$ & 0.8 \\
\hline$>30$ & 414 & $53(12.8)$ & & $0.902(0.516-1.577)$ & 0.717 \\
\hline \multicolumn{6}{|l|}{ Marital Status } \\
\hline Single/divorce & 130 & $28(21.5)$ & 0.131 & & \\
\hline Married/defacto & 1190 & $191(16.3)$ & & & \\
\hline \multicolumn{6}{|l|}{ Literacy } \\
\hline Illiterate & 84 & $18(21.4)$ & 0.252 & & \\
\hline Reads at least a little & 1200 & 199 (16.6) & & & \\
\hline Parity & & & $<0.001$ & & \\
\hline Primiparous & 428 & $105(24.5)$ & & 1 & \\
\hline Multiparous & 802 & $107(13.3)$ & & $0.484(0.347-0.676)$ & $<0.001$ \\
\hline Grand multi & 81 & $8(9.9)$ & & $0.389(0.172-0.883)$ & 0.024 \\
\hline \multicolumn{6}{|l|}{ Pre-eclampsia } \\
\hline Yes & 39 & $14(35.9)$ & & $3.101(1.564-6.150)$ & 0.001 \\
\hline No & 1272 & $206(16.2)$ & & 1 & \\
\hline
\end{tabular}

*Adjusted for pre-eclampsia, age, parity; CI = confidence interval.

\section{Figures}




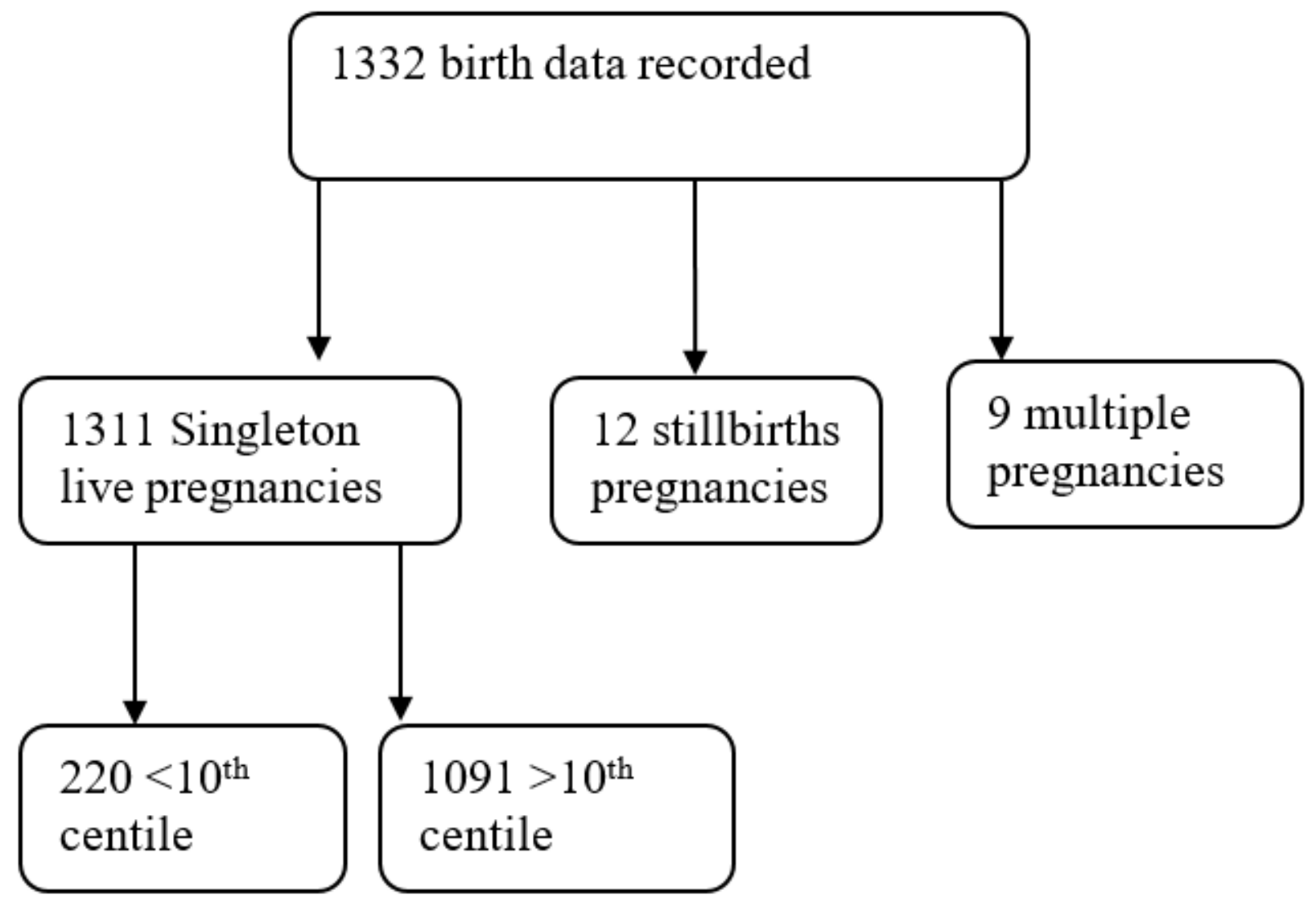

Figure 1

Flow diagram of patients in study

\section{Supplementary Files}

This is a list of supplementary files associated with this preprint. Click to download.

- STROBEchecklistIG21BMCsubmission.docx 